\title{
Venovenous extracorporeal membrane oxygenation for patients with single-ventricle anatomy: A registry report
}

\author{
Tara Karamlou, MD
}

\author{
From the Division of Pediatric Cardiac Surgery, University of California, San Francisco, Benioff Children's \\ Hospital, San Francisco, Calif. \\ Disclosures: Author has nothing to disclose with regard to commercial support. \\ Received for publication March 17, 2016; accepted for publication March 18, 2016. \\ Address for reprints: Tara Karamlou, MD, Division of Pediatric Cardiac Surgery, University of California, \\ San Francisco, Benioff Children's Hospital, Mail Stop 0117550 16th St, 5th Floor, San Francisco, \\ CA 94518 (E-mail: tara.karamlou@ucsf.edu). \\ J Thorac Cardiovasc Surg 2016;151:1446-7 \\ $0022-5223 / \$ 36.00$ \\ Copyright $(2) 2016$ by The American Association for Thoracic Surgery \\ http://dx.doi.org/10.1016/j.jtcvs.2016.03.028
}

The article by Aydin and colleagues ${ }^{1}$ in this issue of the Journal uses the Extracorporeal Life Support (ELSO) registry to describe the growing experience with use of venovenous extracorporeal membrane oxygenation (VV-ECMO) for patients with single-ventricle anatomy (SVA) and pure oxygenation/ventilation impairment. As an investigator who has clearly used registry data frequently over the past 14 years, some may argue that my comments vis-à-vis this article are ironic at best and blatantly hypocritical at worst. This justifiable skepticism notwithstanding, my opinions represent a wealth of lessons learned and an escalating concern that inadequate, incomplete, or biased data, which seem to be increasingly represented in the cardiac surgery literature, may compromise the rigor of contemporary investigation and undermine the validity and import of conclusions reached. A PubMed query ${ }^{2}$ revealed more than 15,288 citations for cardiac registry reports, $59 \%(\mathrm{n}=8933)$ of which were published in the last 5 years. A PubMed search ${ }^{3}$ using the combined terms "descriptive," “database," and "registry" reveals 263 citations. It is within this lens, or conceptual framework, that I offer the following critique.

The descriptive nature of the study, necessitated by the lack of adequate granularity within the chosen dataset, hampers the articulation of a well-defined hypothesis investigating an important knowledge gap in our field. For example, the critical question that I and other reviewers wanted the authors ${ }^{1}$ to address is whether VV-ECMO can be deployed rationally (or at all) among patients with SVA with cardiac failure so that those requiring venoarterial ECMO support could be differentiated outright. The present study reported that among 89 patients with SVA undergoing VV-ECMO within the ELSO database between 1999 and 2012, 30 (32\%) required support for a cardiac indication. Recipients $(\mathrm{N}=4)$ of extracorporeal cardiopulmonary resuscitation were excluded. Unfortunately, although survival was equivalent for those patients with respiratory indications $(51 \%)$ or cardiac indications $(53 \%)$, there is

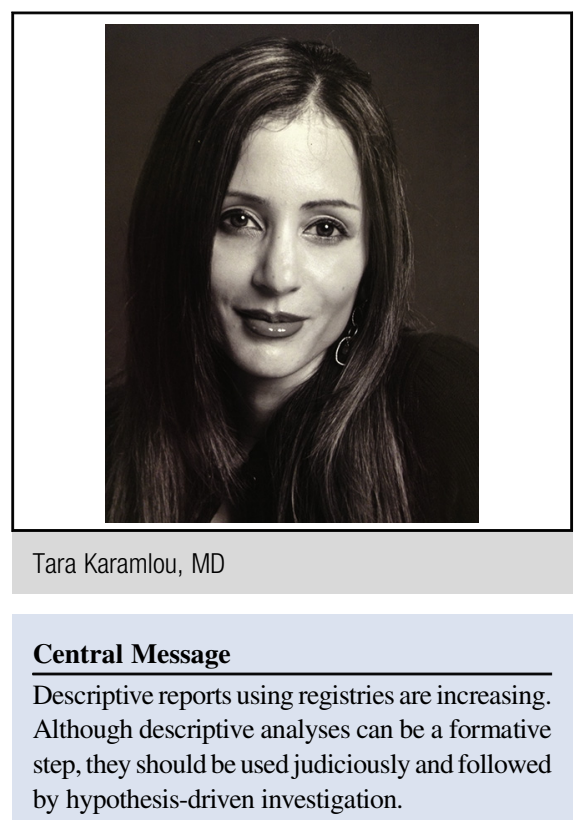

See Article page 1730

See Editorial Commentary page 1737.

no information provided in the article regarding the nature of the cardiac indications. A recently developed cardiac addendum to the existing ELSO registry that ostensibly includes echocardiographic systemic ventricular function, cardiac catheterization data, and so forth is available. However, this addendum reportedly contained data on only $20 \%$ of the study patients, and therefore these data were not included. Moreover, because the registry, as one would expect, contains only de-identified data, there is no mechanism to trace these patients to their respective institutions in the hope of ascertaining additional critical information. Although these limitations are understandable at first and we are tempted to placidly accept them as unsurmountable or beyond the scope of the initial study, one viable option that could significantly improve the article by Adyin and colleagues ${ }^{1}$ would be the inclusion of relevant data and outcomes within one (or better, within all) of the authors' centers. Indeed, that one of the co-authors (R.T.) has published several reports of patients with SVA supported by ECMO underscores the inherent feasibility of such an approach. ${ }^{4-6}$

The lack of data within the ELSO registry within the timeframe studied further compromises the ability of the 
authors ${ }^{1}$ to investigate another critical issue, the timing of ECMO support, both in its relationship to a preceding cardiac procedure and within the context of the entire hospitalization. For example, were these patients immediately postpericardiotomy, were they lingering on a ward, or were they readmitted weeks after their cardiac operation? Nearly every article that investigates outcomes among patients with SVA requiring ECMO support (including the present study) concludes that timely institution of mechanical support is a key principle in improving survival and reducing morbidity. ${ }^{7,8}$ Without knowledge of the context of ECMO deployment, these questions remain unanswered. Moreover, the authors are unable to address the efficacy and utility of relevant, yet contentious, processes of care, such as mandatory in-house extracorporeal cardiopulmonary resuscitation systems; ongoing, regular training (so-called mock codes) for personnel regarding potentially challenging cannulation strategies; mobile ECMO units; and simulation programs for residents and fellows. It is disappointing indeed that additional insight is not provided by the current effort of these highly qualified investigators. At the risk of redundant rhetoric, the inclusion of available institutional clinical data regarding these variables would have supplemented the authors' study significantly.

The statistical analysis used in this article is fairly traditional. Aydin and colleagues ${ }^{1}$ have avoided the pitfall of dichotomizing continuous predictor variables along arbitrary thresholds that may simplify interpretation but limit the generalizability and utility of the statistical information should these thresholds subsequently change over time. The authors also have specified the incremental increase in the odds of mortality associated with changes (either positive or negative) in these continuous predictor variables. It is critical for the reader to understand the magnitude of risk and its direction associated with even small fluctuations within the continuous variables. For example, it is interesting and somewhat counterintuitive that the odds of death increase by 1.16 with an increase in mean arterial blood pressure by $1 \mathrm{~mm} \mathrm{Hg}$.

In the final lexicon, what can we glean from Aydin and colleagues? ${ }^{1}$ Description is an important formative step. There is certainly value in understanding the prevalence of a particular lesion and the outcomes of various treatment strategies to correct these lesions. A registry report provides an opportunity to chronicle limitations (as the authors have nicely done) and therefore iteratively improve the data within the registry. The Society of Thoracic Surgeons Congenital Heart Surgery Database has done this successfully with both consensus and empirically derived database upgrades and the addition of modules designed to focus on specific aspects (ie, anesthesia, perfusion, quality of care processes) that affect surgical outcomes in patients with congenital heart disease. The United
Network Organ Sharing database, in part related to our article that described the deficiencies of coding for congenital heart disease among transplant recipients, has made several additions to their registry. ${ }^{9}$ However, it is now time to transcend these initial steps and invest in the pursuit of the more difficult, yet critically important questions that restrict forward progress in our field. This investment must be concerted and broad-based. Our scientific journals should increasingly insist that a study is hypothesis-driven and that the limitations elucidated by the authors are reasonable and not simply a convenient rationale for avoiding feasible additional data collection or more sophisticated analyses. Potential fruitful avenues on which to focus resources include (1) supporting research infrastructure and dedicated time for interested investigators in our hospitals; (2) using biostatistical input throughout conceptualization, design, and analysis of study data; (3) developing new dataset linkages and exploiting available linkages, which will further streamline the integration of clinical data with existing registry data; and (4) generating incentivized collaboration and increased use of focused consortia to facilitate sharing of scarce or costly resources.

Critics of the descriptive article perhaps can be somewhat mollified by the promise that such initial reports will lead to more guided investigation. Indeed, the authors ${ }^{1}$ mention the availability of more detailed cardiac data within the ELSO registry, which, along with harnessing the aforementioned ideas, may yet give us the answers. Let's hold these authors, and all others, to this promise.

\section{References}

1. Aydin SI, Duffy M, Rodrigues D, Rycus PT, Friedman P, Thiagarajan RR, et al Veno-venous extracorporeal membrane oxygenation for single ventricle patients: a registry report. J Thorac Cardiovasc Surg. 2016;151:1730-6.

2. PubMed.gov. Query using keywords "cardiac registry reports." Available at http://pubmed.ucsf.edu. Accessed March 17, 2016

3. PubMed.gov. Query using combined keywords "descriptive," "database," and "registry." Available at: http://pubmed.ucsf.edu. Accessed March 17, 2016.

4. Roeleveld PP, Wilde RD, Hazekamp M, Rycus PT, Thiagarajan RR. Extracorporeal membrane oxygenation in single ventricle lesions palliated via the hybrid approach. World J Pediatr Congenit Heart Surg. 2014;5:393-7.

5. Jolley M, Thiagarajan RR, Barrett CS, Salvin JW, Cooper DS, Rycus PT, et al Extracorporeal membrane oxygenation in patients undergoing superior cavopulmonary anastomosis. J Thorac Cardiovasc Surg. 2014;148:1512-8.

6. Allan CK, Thiagarajan RR, del Nido PJ, Roth SJ, Almodovar MC, Laussen PC. Indication for initiation of mechanical circulatory support impacts survival of infants with shunted single-ventricle physiology supported with extracorporeal membrane oxygenation. J Thorac Cardiovasc Surg. 2007;133:600-7.

7. Alsoufi BA, Awan A, Manlhiot C, Al-Halees Z, Al-Ahmadi M, McCrindle BW et al. Does single ventricle physiology affect survival of children requiring extracorporeal membrane oxygenation support following cardiac surgery? World J Pediatr Congenit Heart Surg. 2014;5:7-15.

8. Duncan BW, Ibrahim AE, Hraska V, del Nido PJ, Laussen PC, Wessel DL, et al Use of rapid-deployment extracorporeal membrane oxygenation for the resuscitation of pediatric patients with heart disease after cardiac arrest. J Thorac Cardiovasc Surg. 1998;116:305-11.

9. Karamlou T, Hirsch J, Welke K, Ohye RG, Bove EL, Devaney EJ, et al. A United Network for Organ Sharing analysis of heart transplantation in adults with congenital heart disease: outcomes and factors associated with mortality and retransplantation. J Thorac Cardiovasc Surg. 2010;140:161-8. 